\title{
Detección de factores de riesgo en los trastornos del desarrollo en preescolares ${ }^{1}$
}

\author{
Lucía Arciniega Buenrostro² \\ María Elena Márquez Caraveo ${ }^{3}$ \\ Isaura Díaz Mayer-Goyenechea
}

doi:10.11144/Javeriana.IE16-2.dfdr

Cómo citar: Arciniega Buenrostro L, Márquez Caraveo ME, Díaz Mayer-Goyenechea I. Detección de factores de riesgo en los trastornos del desarrollo en preescolares. Investig Enferm. Imagen Desarr. 2014;16(2):95-113. doi:10.11144/Javeriana.IE16-2.dfdr

1. Artículo de investigación. Recibido: 6 de marzo de 2014. Revisado: 12 y 21 de mayo de 2014. Aprobado: 9 de junio de 2014.

2. Magistra en Enfermería adscrita a la División de Investigación del Hospital Psiquiátrico Infantil Dr. Juan N. Navarro, Secretaría de Salud, México. Correo electrónico: arciniegabl@yahoo.com.mx

3. Doctora en Psiquiatra Infantil y de la Adolescencia. Jefa de la División de Investigación del Hospital Psiquiátrico Infantil Dr. Juan N. Navarro, Secretaría de Salud, México. Correo electrónico: malenamarquezc@gmail.com

4. Licenciada psicóloga adscrita a la División de Investigación del Hospital Psiquiátrico Infantil Dr. Juan N. Navarro, Secretaría de Salud, México. Correo electrónico: psic.isauradiaz@gmail.com 


\section{Resumen}

Introducción: Los primeros años de vida constituyen un periodo crítico del desarrollo humano, y la identificación de los factores biológicos y psicosociales que lo impactan es crucial para la prevención e intervención preventiva de los trastornos asociados. Metodología: Se estudiaron 70 madres de preescolares: 35 de una guardería y 35 de un hospital psiquiátrico, ambos en Ciudad de México, en 2009. Los factores de riesgo se identificaron con el informe de las madres, quienes contestaron el instrumento de identificación de factores de riesgo y protección de Leckman sobre la etapa prenatal, parto y alumbramiento, primeros cinco años de vida e historia pediátrica. El diseño del estudio fue retrospectivo/prospectivo, transversal y comparativo. El análisis estadístico se realizó con la prueba T de Student para muestras independientes. Resultados: Se encontraron diferencias significativas en la dimensión biológico-individual $(t=-3,027 ; g 1: 68 ; p=0,000)$ y en la contextual $(t=-1,111 ; g 1: 66 ; p=0,839)$, es decir, el $78,6 \%$ del grupo clínico tiene factores de riesgo en este periodo del desarrollo, en contraste con el grupo de comunidad, cuyos factores de riesgo se centraron en la dimensión parental ( $\mathrm{t}=0,915 ; \mathrm{gl}: 66 ; \mathrm{p}=0,839)$. Conclusiones: Es importante identificar los factores de riesgo biológico y psicosociales que inciden en el desarrollo infantil, a fin de que el profesional de la salud ponga énfasis en la prevención más que en la rehabilitación de los trastornos.

Palabras clave: factores de riesgo; trastornos del desarrollo infantil; salud mental

\section{Detection of Risk Factors in Developmental Disorders in Preschool}

\section{Abstract}

Introduction: The early years are a critical period of human development, and identification of biological and psychosocial factors that impact it is crucial for preventive intervention and prevention of associated disorders. Methodology: 70 mothers of preschool children were studied. 35 from a nursery and 35 from a psychiatric hospital, both in Mexico City in 2009. Risk factors were identified in the report of mothers who answered the instrument to identify risk factors and protection by Leckman on prenatal stage, labor and delivery, first five years of life and pediatric history. The study design was retrospective / prospective, cross-sectional and comparative. Statistical analysis was performed using Student's $\mathrm{T}$ test for independent samples. Results: There were found significant differences in the biological-individual dimension $(\mathrm{t}=$ -3.027, df: 68; $\mathrm{p}=0.000$ ) and in the context $(\mathrm{t}=-1.111$, df: $66 ; \mathrm{p}=0.839)$, i.e., $78,6 \%$ of the clinical group have risk factors in this period of development, in contrast to the community group, whose risk factors focused on parental dimension $(t=0.915$, df: $66 ; p=0.839$ ). Conclusions: It is important to identify the biological and psychosocial risk factors that affect child development, so that the health professional makes emphasis on prevention rather than rehabilitation of disorders

Keywords: risk factors; developmental disorders in children; mental health 


\section{Detecção de fatores de risco em transtornos globais do desenvolvimento em crianças de pré-escolar}

\section{Resumo}

Introdução: Os primeiros anos de vida constituem um período crítico do desenvolvimento humano e a identificação dos fatores biológicos e psicossociais que o impactam é crucial para a prevenção e intervenção preventiva dos transtornos associados. Metodologia: Estudaram-se 70 mães de crianças de pré-escolar: 35 de uma creche e 35 de um hospital psiquiátrico, ambos os dois grupos em Cidade de México, em 2009. Os fatores de risco foram identificados com o relatório das mães, quem contestaram o instrumento de identificação de fatores de risco e proteção de Leckman sobre a etapa pré-natal, parto e nascimento, primeiros cinco anos de vida e história pediátrica. O desenho do estudo foi retrospetivo/prospetivo, transversal e comparativo. A análise estadística foi realizada com a proba T de Student para amostras independentes. Resultados: Encontraram-se diferenças significativas na dimensão biológica-individual ( $\mathrm{t}=-3,027$; $\mathrm{gl}$ : 68; $\mathrm{p}=0,000)$ e na contextual $(\mathrm{t}=$ $-1,111$; gl: $66 ; \mathrm{p}=0,839$ ), ou seja, o $78,6 \%$ da turma clínica têm fatores de risco neste período de desenvolvimento, em contraste com a turma de comunidade, cujos fatores de risco foram centrados na dimensão parental ( $t=0,915$; gl: $66 ; p=0,839$ ). Conclusões: É importante identificar os fatores de risco biológico e psicossociais que incidem no desenvolvimento infantil, a fim do profissional da saúde remarcar a prevenção mais do que na reabilitação dos transtornos.

Palavras chave: fatores de risco; transtornos globais do desenvolvimento infantil; saúde mental 


\section{Antecedentes de investigación}

Diversos estudios acerca de factores de riesgo y protección en el desarrollo infantil (1-5) coinciden con los modelos contemporáneos del desarrollo infantil (6-9) que explican la relación con las interacciones dinámicas continuas entre el niño y la experiencia provista por la familia y el contexto social. Hoy día se reconoce el rol que los factores de riesgo desempeñan en el desarrollo de la psicopatología infantil, de acuerdo con el tiempo de exposición a uno o a varios de estos factores $(10,11)$.

Los factores se identifican a partir de los resultados de diversas investigaciones y se pueden dividir para su estudio en las siguientes dimensiones: biológico-individual (prenatal, perinatal y posnatal) parental y contextual. Se hace hincapié en la visión de riesgo de los factores biológicoindividuales. Algunos estudios internacionales y nacionales encontraron una correlación altamente significativa entre el tipo de trastorno y la edad de los sujetos, y ello mostró que la psicopatología infantil sigue una pauta evolutiva. También se encontró una correlación significativa entre el tipo de trastorno y el género (12).

Dentro de estos se encuentran los trastornos genéticos, como la discapacidad intelectual, donde una tercera parte es de causa genética, otra tercera por complicaciones perinatales y en el resto de la población se desconoce la causa. Esta condición se caracteriza por la presencia de un cociente intelectual menor a 70, una limitación adaptativa (como las habilidades de la vida cotidiana, la comunicación y la socialización) e inicio antes de los 18 años de edad. En el ámbito mundial se señala que la discapacidad intelectual es frecuente, con un alto impacto en el funcionamiento individual y una prevalencia aproximada del 1-4\%. Respecto a los factores de riesgo en Latinoamérica, la prevalencia puede ser cuatro veces mayor, por su asociación con factores como la desnutrición, las complicaciones obstétricas y perinatales, la prematurez, la intoxicación por plomo, las infecciones del sistema nervioso central y la pobreza, que han sido superados en países desarrollados (13).

La prevalencia exacta en México se desconoce, por la ausencia de investigación epidemiológica. Solo se tienen los reportes oficiales sobre la discapacidad, que provienen del Censo de Población y Vivienda y que no representan una medición diagnóstica, pero aportan algunos datos al respecto (13). El censo de 2010 contempló un cuestionario ampliado donde se reporta la población que presenta alguna discapacidad, vista desde el enfoque de limitaciones en la actividad. Es decir, se contaron las personas con alguna limitación para atender el cuidado personal, para caminar y moverse, para escuchar, para hablar o comunicarse, para poner atención, para ver, y con alguna limitación mental.

De acuerdo con este informe, la discapacidad alcanzó el 5,1\% de la población total, es decir, 5.739.270 mexicanos. La detección constituye un reto en nuestro país, ya que el retraso en la atención especializada es común para los trastornos psiquiátricos.

En uno de los pocos estudios realizados en México en población infantil, se estimaron hasta 8 años para la atención del retraso mental. Se 
documentó la búsqueda de ayuda en un $13 \%$. El profesionista contactado fue el psicólogo en el 66\%, y un médico general, en el $28 \%$ de los casos (14).

La detección de la discapacidad intelectual consiste en la identificación de niños en riesgo de algún tipo de desarrollo anormal, con énfasis en el sondeo del lenguaje, combinado con herramientas como los instrumentos de tamizaje del desarrollo psicométricamente fiables (15).

También existe asociación entre problemas biológico-individuales (pre, peri y posnatales) y problemas de psicopatología infantil. Se señala que con problemas en el parto, alcoholismo familiar, enfermedades frecuentes, estado psicopatológico de la madre a los trastornos depresivos, tener un padre o madre joven o ser varón se asocia con los trastornos conductuales y de ansiedad. El temperamento puede ser visto como una complicación biológica (genética) de riesgo, ya que los niños que tienden a reaccionar de forma negativa ante situaciones que les generan estrés es decir, son niños que regularmente gritan o avientan cosas cuando se enojan, quienes experimentan con mayor frecuencia emociones negativas, junto con una falta de concentración para concluir sus tareas- presentaron algún trastorno de tipo interiorizado $(12,16-18)$.

Dentro de los factores de riesgo parentales, la psicopatología familiar y la depresión materna generan riesgo general para desarrollar psicopatología entre los padres e hijos de tres generaciones de 2,5 a 2,7 veces más que la población en general $(7,19,20)$.

Los ambientes de crianza tienen efectos importantes en una variedad de desenlaces que incluyen desde variaciones en el funcionamiento adaptativo, pasando por el desarrollo psicológico saludable y el éxito en la escuela, hasta desenlaces psicopatológicos como el uso y abuso de drogas, el comportamiento agresivo y problemas de ansiedad en niños y adolescentes (21).

Factores de riesgo contextuales, como la familia, los problemas económicos, la baja educación materna, los conflictos entre los padres, las enfermedades crónicas en algún miembro familiar, la falta de recursos sociales, las familias monoparentales, el escaso apoyo emocional y la influencia escolar son estresores que influyen negativa o positivamente en la salud mental y en el funcionamiento psicosocial de niños y adolescentes $(22,23)$.

\section{Objetivo general}

Detectar factores de riesgo en dos grupos de niños preescolares con problemas del desarrollo y sin estos con el instrumento de identificación de factores de riesgo y protección de Leckman (24).

\section{Objetivos especificos}

1. Describir los aspectos sociodemográficos de ambos grupos.

2. Comparar la presencia de factores de riesgo biológico-individuales (pre, peri y posnatales), parental y contextual en el grupo de comunidad frente al grupo clínico (con trastornos del desarrollo).

3. Comparar la presencia de factores de riesgo por nivel socioeconómico. 


\section{Material y método}

Se trató de un estudio retrospectivo/prospectivo, transversal y comparativo. Se incluyeron 70 niños preescolares de 3 a 5 años de edad, 35 que asistian a una guardería oficial y 35 pacientes de un hospital psiquiátrico infantil de la Ciudad de México, cuyas madres aceptaron participar en el estudio

\section{Instrumento de identificación de factores de riesgo y protección modificado}

Este instrumento fue desarrollado en la Universidad de Yale de forma estructurada, como autorreporte para padres de pacientes con sindrome de Tourette, llamado originalmente Modified Schedule for Risk and Protective Factors (MSRPF) $(3,4,25)$. Ha sido utilizado en diferentes estudios longitudinales y epidemiológicos, además de estudios genéticos familiares, dentro de los cuales están el de Santangelo y Vasconcelos $(3,4)$.

El instrumento original estaba conformado por 23 páginas con 73 preguntas de estructura diferente: tipo Likert, abierta, semiestructurada y dicotómica. El instrumento recopila información de cinco dimensiones: etapa prenatal, parto y alumbramiento, del nacimiento al primer año de vida, del año a los cinco años y una historia pediátrica.

\section{Proceso de validación del instrumento}

- Traducción/retraducción y piloteo del instrumento, cuidando los aspectos culturales de la población donde se aplicaría y que el lenguaje fuera claro.

- Las preguntas abiertas se codificaron en rangos, por ejemplo: ¿qué edad tenía cuando usted se embarazó? 1: < 20 años; 2: $>20$ años.

- La calificación de todas las respuestas se cambió a dicotómica, donde: 1 = sí y 2 = no; sí = con riesgo y no = sin riesgo (engloba las respuestas: normal, algunas veces y nunca).

- La nueva versión utilizada en este estudio fue de 66 preguntas, con respuestas dicotómicas y los factores de riesgo se agruparon en 5 dimensiones resultado del análisis factorial y como lo propone la Organización Mundial de la Salud: biológico-individual (prenatal, perinatal y posnatal), parental y contextual.

Este instrumento identifica los factores de riesgo para la salud mental y el desarrollo infantil óptimo en niños de edad preescolar a partir del informe de los padres, e informa sobre aspectos del embarazo, parto, nacimiento y primeros cinco años de vida de sus hijos. 


\section{Procedimiento}

Se obtuvo el permiso del autor del instrumento (Leckman) para su utilización, así como la aprobación del comité de investigación del Hospital Psiquiátrico Infantil Dr. Juan N. Navarro, y de la directora de una guardería de la Secretaría de Desarrollo Social. Los padres de familia de ambos grupos (comunidad $=35$ y clínico $=35$ ) firmaron la carta de consentimiento después de conocer la descripción del estudio, donde se recalcó la importancia de la identificación temprana de factores de riesgo para el desarrollo infantil.

\section{Consideraciones éticas}

Este estudio cumple con las normas del acta de Helsinki y Nüremberg para la investigación en seres humanos. De igual manera, el proyecto de investigación fue aprobado por el comité de investigación del Hospital Psiquiátrico Infantil Dr. Juan N. Navarro.

\section{Análisis estadístico}

Con el fin de evaluar el nivel de riesgo, se asignaron niveles de 0 a 3, los cuales corresponden al siguiente porcentaje: < $15 \%$ (nivel 0): muy bajo riesgo (MBR); 16-30\% (nivel 1): bajo riesgo (BR); 31-50\% (nivel 2): alto riesgo (AR), y > 50\% (nivel 3): muy alto riesgo (MAR).

El análisis se realizó por medio de estadística descriptiva para las variables sociodemográficas y las puntuaciones del instrumento. Con el programa SPSS versión 17 se analizaron las diferencias en las medias de las puntuaciones del instrumento, mediante la prueba T de Student. E1 nivel de significancia estadística se estableció con una $\mathrm{p}<0,05$.

\section{Resultados}

Participaron un total de 70 preescolares, divididos en dos grupos. En la tabla 1 se observa que el grupo 1, identificado como "de comunidad" (G1COM), incluyó a 35 niños, de los cuales 21 (60\%), fueron varones y 14 (40\%) fueron mujeres. En el grupo 2, llamado “clínico" (G2CLIN), agrupó a 35 niños, de los cuales 26 (74\%) fueron varones y $9(26 \%)$ correspondieron a participantes del sexo femenino. Respecto a la edad, se observa que la media de edad en los preescolares del G1COM fue discretamente menor $(\mu=3,69 ; \mathrm{DE}=0,796)$ a la del G2CLIN $(\mu=4,34 ; \mathrm{DE}=0,838)$. En el caso de las madres se puede observar que la media de edad es similar en ambos grupos y que, en general, las madres son más jóvenes que los padres en ambos grupos. Por otro lado, en la variable de escolaridad se reportó un predominio del nivel de preparatoria y nivel técnico en el G1COM, a diferencia del G2CLIN, en que predominó el nivel de secundaria. 


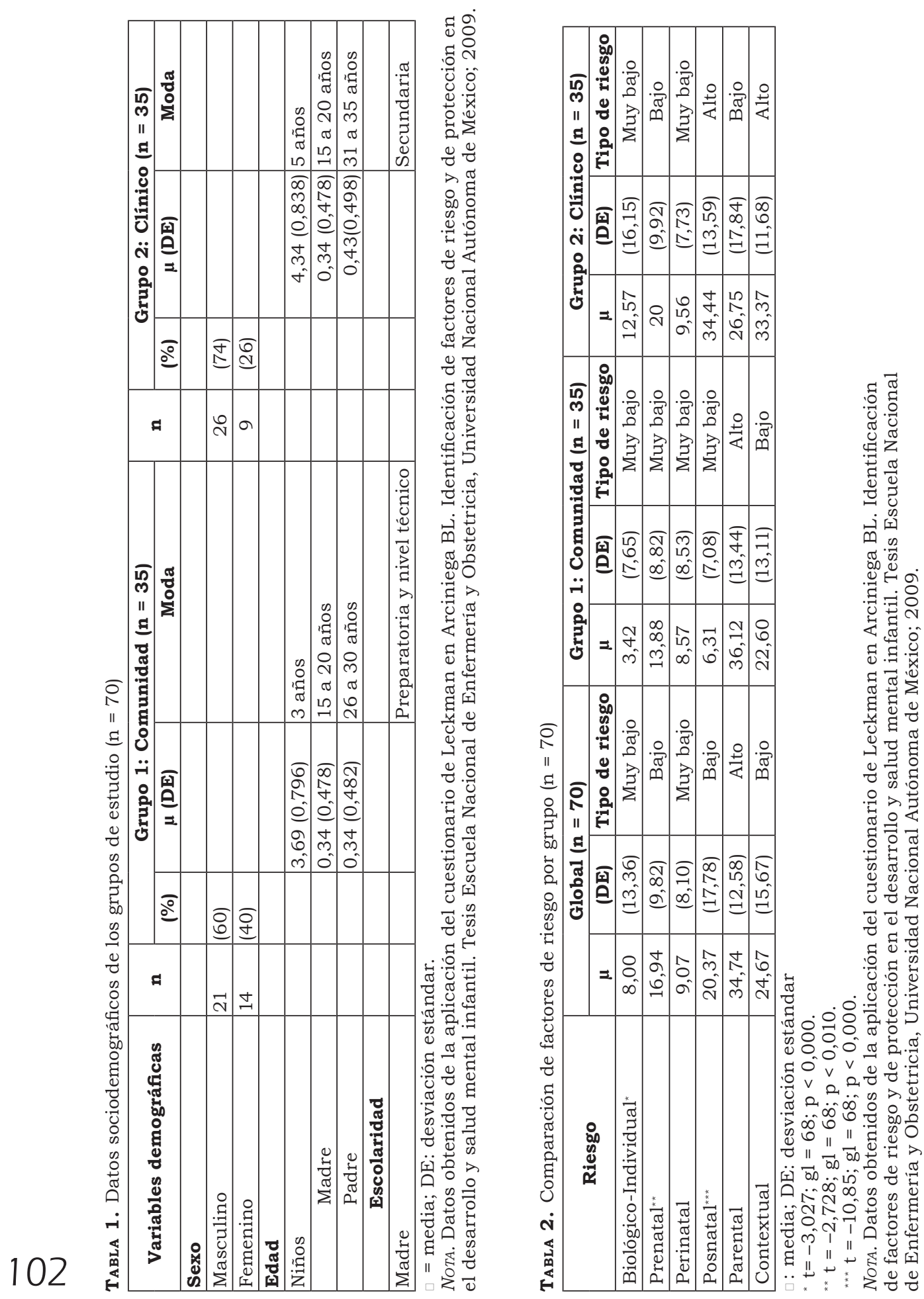


En la tabla 2 puede observarse que en el conjunto de la muestra se identificaron riesgos de tipo biológico individual, parental y contextual. Solo los factores de riesgo parental registraron puntuaciones porcentuales correspondientes a nivel alto (AR). Sin embargo, al realizar el análisis de los factores de riesgo entre los grupos, se observan diferencias entre los grupos G1COM y G2CLIN. Así, mientras que en el G1COM predominan los riesgos muy bajos (MBR) o bajos (BR), en los planos biológico-individual y contextual, el riesgo de tipo parental exhibió las más altas puntaciones (AR). En contraste, en G2CLIN se identificaron a nivel alto (RA) factores de tipo biológico individual, específicamente los de tipo prenatal y posnatal, y los factores de riesgo de tipo contextual.

El análisis por nivel socioeconómico se reporta en la tabla 3, donde es posible observar que las personas con bajo nivel de ingresos tienen una diferencia significativa de factores de riesgo, en contraste con las de suficientes ingresos.

Tabla 3. Comparación de factores de riesgo por estatus socioeconómico (alto y bajo)

\begin{tabular}{|l|l|l|l|c|}
\hline \multicolumn{2}{|c|}{ Factor de riesgo: estatus socioeconómico } & $\mathbf{n}$ & $\boldsymbol{\mu}$ & DE \\
\hline \multirow{2}{*}{$\mathrm{d}$} & Bajo nivel de ingresos & 60 & $26^{* * *}$ & 15,590 \\
\cline { 2 - 5 } & Suficiente nivel de ingresos & 10 & 11 & 7,799 \\
\hline
\end{tabular}

$\square$ : media; DE: desviación estándar.

${ }^{* * *} \mathrm{t}=3,072 ; \mathrm{gl}=68 ; \mathrm{p}>0,003$

Nota. Datos obtenidos de la aplicación del cuestionario de Leckman en Arciniega BL. Identificación de factores de riesgo y de protección en el desarrollo y salud mental infantil. Tesis Escuela Nacional de Enfermería y Obstetricia, Universidad Nacional Autónoma de México; 2009.

\section{Discusión}

En la presente investigación se observó que los varones constituyeron casi las tres cuartas partes del grupo clínico. Estos resultados son similares con lo que otros autores reportan (14), en cuanto a que los niños son más vulnerables a tener problemas externalizantes: agresión, hiperactividad y trastornos de conducta, además de trastornos del desarrollo: discapacidad intelectual, autismo, lenguaje, aprendizaje, lectoescritura y problemas de identificación sexual en la infancia temprana. Estos datos concuerdan con la evidencia encontrada por otros autores $(23,24)$, que señalan que los varones son más vulnerables. Esto traduce lo urgente de diseñar programas de intervención preventiva de tipo selectiva o indicada (2). La literatura señala que la demanda de atención médica es dos veces mayor en los varones que en las niñas. El modelo de prevención universal (a toda la población), selectiva (población en riesgo) o indicada (población con problemas menores) (26) surge como alternativa al modelo de prevención primaria, secundaria y terciaria, dado que la génesis, en el caso de las enfermedades mentales, 
tiene origen de acuerdo con la etapa del desarrollo en la que se encuentre el individuo y a partir de la interacción de las características particulares individuales, el ambiente y la cultura. A partir de los datos de esta investigación, se considera importante llevar a cabo acciones que se centren en los varones como un subgrupo en riesgo de presentar problemas del desarrollo en centros de salud, centros comunitarios de salud mental, hospital pediátrico o unidad materno-infantil, etc., en los que el personal de enfermería tiene un papel prioritario en el diseño de intervenciones diferenciales, dependiendo del nivel de atención a la salud en el que se trabaje.

Sin embargo, también hay autores que refieren que en las niñas pueden estar presentes, aunque en menor grado, estos problemas y no ser tan evidentes como en los niños, cuestión que se revierte en la adolescencia, cuando las jóvenes reflejan problemas internalizantes, como depresión e intento suicida. Respecto a la edad, es posible que la media discretamente mayor en el grupo clínico $v$ s. el de comunidad se explique por el hecho de que el grupo de comunidad corresponde a preescolares que asisten a una sistema de cuidado infantil (guardería); mientras que el clínico es aquel que busca atención médica por un problema del desarrollo, quizá evidente a más tardía edad. También llama la atención que en el grupo clínico las madres exhiben un nivel de escolaridad más bajo.

Respecto a la presencia de factores de riesgo, se destaca que los hay en ambos grupos. Así, aunque más de un factor de riesgo evaluado como alto se observa en el grupo clínico, el grupo de la comunidad (G1COM) presenta más altas puntuaciones en el rubro de riesgo parental. Lo anterior hace suponer factores como psicopatología parental o problemas de crianza que no se están atendiendo en el ámbito comunitario. Aun cuando no se midió directamente la salud mental de los padres, sería importante descartar la posible existencia de psicopatología familiar, particularmente depresión. Estos datos son preocupantes, pues aunque los niños no exhiben patología clínica, están en condición de riesgo y vulnerabilidad para el desarrollo de problemas diversos.

Es importante que los modelos de atención clínica incluyan la perspectiva de riesgo en sus intervenciones. La enfermera y todo profesional de la salud en las instituciones de atención a la comunidad (primer y segundo nivel de atención) tienen un papel relevante en las acciones preventivas universales (a toda la población) y selectivas (grupos de riesgo). La prevención universal incluiría, a partir de los datos obtenidos en este estudio, la promoción de la salud mental en los futuros padres en el ámbito comunitario (familia, escuela, albergues infantiles y organizaciones no gubernamentales) y la prevención selectiva mediante la psicoeducación a la población eventualmente en riesgo que asiste a clínicas y hospitales en la detección, diagnóstico y tratamiento de problemas detectados en este estudio como ansiedad, depresión, abuso de sustancias, discordia marital $\mathrm{y}$ violencia familiar en de clínicas y hospitales.

En cuanto al G2CLIN, los índices más altos de riesgo que se observan son en los factores de tipo biológico individual, específicamente el posnatal y el contextual. Ambos datos coinciden con lo reportado en la 
literatura, ya que diversos autores (14) resaltan la importancia de estas contribuciones biológicas en los problemas del desarrollo. Estos factores incluyen el coeficiente intelectual bajo o el temperamento difícil, factores adversos al desarrollo infantil óptimo en los primeros años de vida. Estos datos son reforzados por los hallazgos de otros autores, quienes sostienen que el cociente intelectual de los niños guarda relación con otras variables, como el comportamiento de los padres, la salud mental, el grado de ansiedad, la educación, las creencias de los padres acerca del desarrollo del niño, el tamaño de la familia, el estrés de sucesos vitales, la ocupación de los padres y la desventaja de las minorías. Además, el temperamento se correlaciona positivamente, sobre todo en las niñas con trastornos internalizantes, como ansiedad y depresión.

Lo anterior coincide con los reportado por autores (19-22) que sostienen que la probabilidad de presentar psicopatología es entre dos y tres veces mayor cuando hay trastornos psiquiátricos en los padres y que el riesgo general para desarrollar psicopatología familiar y depresión obtenida entre los padres e hijos de tres generaciones es de 2,5 a 2,7 veces, lo cual los coloca en un nivel II de alto riesgo, para presentar trastornos emocionales y conductuales como estrés, ansiedad y depresión en los niños. En este sentido, en el Hospital Psiquiátrico Infantil, la depresión es el primer diagnóstico en las niñas.

De acuerdo con recomendaciones internacionales, la organización óptima de servicios de salud mental para niños y adolescentes (2) incluye equipos multidisciplinarios con participación del personal de enfermería en los centros comunitarios de salud mental y en hospitales pediátricos que proporcionen tratamiento de los problemas del desarrollo y la salud mental de los niños y sus familias, al igual que proporcionen asesoría a los centros de salud generales y lleven a cabo investigación de los casos referidos al ámbito comunitario.

Finalmente, es importante resaltar que el factor de riesgo contextual exhibió también un nivel alto en el grupo clínico. Esta dimensión incluye pobreza, baja educación materna, falta de recursos sociales, bajo soporte emocional y aspectos de escolarización. El análisis tuvo en cuenta la separación por nivel de ingresos con el propósito de evaluar si las familias de más bajo ingreso presentan mayor número de factores de riesgo.

Los datos obtenidos concuerdan con lo encontrado dentro de la literatura revisada $(21,23)$, donde el entorno familiar en condiciones de pobreza se caracteriza por la presente de una importante fuente de acontecimientos, tanto vitales como cotidianos, que suponen riesgos acumulativos para el saludable desarrollo de los niños (11). En este sentido, existe evidencia de que los estresores dentro de la familia pueden influir negativamente o positivamente en la salud mental y en el funcionamiento psicosocial de niños y adolescentes. Se ha encontrado que los estresores familiares más estudiados son los siguientes: problemas económicos, conflictos entre los padres, familias monoparentales, enfermedades crónicas en algún miembro familiar, estados depresivos en alguno de los padres, divorcio o separación, muerte de algún familiar cercano y relaciones padres-hijos conflictivas. Esta 
combinación de factores contextuales se debe tomar en cuenta de manera conjunta con otros de índole biológico-individual, parental y familiar, ya que la persistencia de uno o la combinación de varios tendrán impacto en el desarrollo y salud mental del niño, lo que es esperable, dado que los problemas del desarrollo están mayormente influidos por este tipo de patrones $(27,28)$.

\section{Comparación de factores de riesgo biológico-individual (prenatales y posnatales)}

Mediante la aplicación de la prueba T de Student se compararon los resultados de los factores de riesgo por grupos de estudio. Lo anterior se fundamentó en que, de acuerdo con la literatura, las condiciones de mayor riesgo incluyen tanto factores biológicos como psicosociales. Estos incluyen el nacimiento prematuro, la enfermedad médica seria, el temperamento infantil, el apego infante-cuidador, los padres, el estado civil, las interacciones con su medio ambiente, la clase social, la pobreza, la paternidad adolescente y la violencia familiar. Por lo tanto, es importante documentar cuáles de estos factores se encuentran predominantemente en un grupo con problemas del desarrollo y si existen algunos de ellos en grupos de niños aparentemente sanos. Se sabe que estos factores interactúan y afectan el desarrollo infantil durante los tres primeros años de vida o después de estos.

El resultado se aprecia mejor en el contexto de los niños que acuden a consulta en el hospital psiquiátrico por diversos problemas, entre ellos retraso en el desarrollo, discapacidad intelectual, etc., lo cual explica que sea el grupo en el cual hubo mayor significancia. Estos resultados también coincidieron con los datos obtenidos por otros grupos de investigadores (10-15,17), entre los cuales se afirma que el comportamiento de los niños preescolares está altamente afectado por variables que se suman de las dimensiones estudiadas, por ejemplo, la identificación de factores de riesgo biológico (pre y posnatal), tanto como el contextual (pobreza), que se considerará más específicamente (29).

\section{Comparación de la presencia de factores de riesgo por nivel socioeconómico en ambos grupos}

Los factores de riesgo parentales están asociados a problemas conductuales y trastornos psiquiátricos en los niños. Muchos son resultado de múltiples circunstancias familiares adversas crónicas que caracterizan o se exacerban en los ambientes de pobreza. Por ejemplo, la enfermedad mental de uno de los padres, la depresión materna, la discordia marital, la muerte de un padre, el divorcio o las familias reconstituidas, uniparentales o numerosas. La literatura sobre el tema reporta que muchas de estas familias exhiben patrones de crianza caracterizados por uso de castigo frecuente, abandono emocional, cambios de escuela y casa, padres migrantes, violencia familiar, necesidad de asistencia social y cuidado institucional del niño $(24,30)$. 
La influencia de otro factor de riesgo, como la uniparentalidad (madres solteras o padres solteros), afecta las posibilidades de supervisión de los hijos, lo que a su vez se relaciona con la calidad de la red de apoyo con que cuente la madre (3). Asimismo, la pobreza está asociada con una mayor tasa de factores de riesgo. Hoy se sabe que no es la existencia de un solo factor de riesgo lo que puede determinar patología, sino la concurrencia de varios factores de riesgo (8). Por ejemplo, un estudio encontró que hay una relación significativa entre la suma de acontecimientos vitales estresantes de tipo parental y contextual que impactan el desarrollo temprano y posterior de un individuo, como la aparición de trastornos de ansiedad, donde la presencia de psicopatología en los padres, el temperamento del niño, antecedentes familiares de psicopatología y el nivel socioeconómico bajo facilitan la aparición de determinados problemas en el embarazo, parto o desarrollo.

Los resultados de esta investigación coincidieron con los datos obtenidos por otros grupos de investigadores $(21,23)$, los cuales afirman que el comportamiento de los niños preescolares está altamente afectado por variables del contexto social. Lo anterior es consistente con un estudio longitudinal de 18.000 familias con niños pequeños que forman parte del Millennium Cohort Study, donde se analizaron 10 factores de riesgo como: vivir en condiciones de hacinamiento; tener una madre adolescente, uno o más padres con depresión, una discapacidad física, escasa formación escolar; abusar de sustancias, o padecer de consumo excesivo de alcohol, estrés financiero, falta de trabajo y violencia doméstica. Se explica que el bajo nivel socioeconómico tiene asociación con todas las dimensiones de salud del niño, ya que las personas de clases sociales más bajas poseen un mayor riesgo de contraer enfermedades graves que limitan su capacidad física y mental y no llegan a su pleno desarrollo, por lo que son empleados a largo plazo en oficios manuales no calificados y mal asalariados en la vida adulta, lo que se torna una cadena nociva en las futuras generaciones, además de tener mayor probabilidad de ser diagnosticados con problemas de salud mental en la niñez.

Entre los resultados de esta línea de trabajo también se encontró que de los 10 riesgos mencionados, el $28 \%$ de las familias con niños pequeños en el Reino Unido en el 2001 se enfrentaba a 2 o más de estos riesgos, lo que constituye un estimado de 192.000 niños de corta edad (menores de un año de edad). Lo anterior quiere decir que el impacto de estos factores de riesgo en los resultados del desarrollo es que los problemas de conducta se incrementan en los niños que viven en familias con múltiples riesgos, entre ellos aquellos quienes viven en hogares de bajos ingresos. Este dato concuerda con el reportado en el Centro Nacional para Niños en la Pobreza, que refiere que el $79 \%$ de los niños con problemas de salud mental tienen necesidades insatisfechas.

Ante estos resultados encontrados, el profesional de salud debe enfocar su atención en la capacitación en la problemática psicosocial en cada nivel de intervención para el niño y el adolescente, donde la intervención de enfermería ideal recomendada por los organismos internacionales de salud deberá estar centrada en el primer nivel y centros de salud mental (2). 
La literatura refiere que las intervenciones deben estar formuladas con base en la mejor evidencia disponible, en el contexto de cada país, por lo que - de acuerdo con los datos obtenidos- es importante llevar a cabo investigación en distintos grupos, a fin de caracterizar los riesgos existentes y diseñar intervenciones específicas, basadas en datos de investigación.

Lo anterior favorecerá la mejora en la efectividad, seguridad y calidad de la atención médica y contribuirá, de esta manera, al bienestar de las personas y de las comunidades, el cual constituye el objetivo central y la razón de ser de los servicios de salud (26).

Con el fin de mejorar el desarrollo del niño, la enfermera puede salir del ámbito clínico hacia la comunidad, a fin de desarrollar programas de seguimiento y adhesión al tratamiento, siendo una educadora y promotora para padres, familiares y pacientes, como lo sugiere la Organización Mundial de la Salud (OMS).

En este sentido, la atención primaria debe de ser el punto donde se articule la atención de la salud mental comunitaria, ya que el número total de recursos humanos que trabajan en dispositivos de salud mental asciende a 10.000 y la tasa por cada 100.000 habitantes se distribuye de la siguiente manera: 1,6 psiquiatras, 1 médico, 3 enfermeras, 1 psicólogo, 0,53 trabajadores sociales, 0,19 terapeutas y 2 profesionales/técnicos de salud. Estos recursos son insuficientes, mal distribuidos y la mayoría de ellos están concentrados en los hospitales psiquiátricos (31).

La enfermera domiciliaria tendrá el compromiso y la necesidad de impactar en la comunidad en las siguientes aéreas de oportunidad:

1. Promover el desarrollo de logros de los niños y sus habilidades cognitivas.

2. Fortalecer un vínculo seguro entre el niño y los padres.

3. Impulsar positivamente los roles parentales positivos y la comunicación.

4. Mejorar el nivel educativo de los padres.

Algunas de las estrategias de intervención mencionadas por O'Connell y cols. (32) en la revisión del Instituto de Medicina son las visitas domiciliarias que tengan como meta embarazos exitosos y el desarrollo adecuado del niño durante los primeros años de vida (32).

\section{Intervenciones preventivas específicas}

Prevención de embarazo en adolescentes: especialmente en menores de 16 años de edad, ya que es un factor de riesgo para parto pretérmino, retardo en el crecimiento intrauterino y complicaciones perinatales. Aumento de la probabilidad de ser madre soltera, con bajo nivel educativo e ingreso mensual, situación que sugiere un riesgo acumulado para el desarrollo infantil (33).

Prevención de problemas de conducta y emocionales: favorecerán que cuando el niño sea adulto goce de una salud mental óptima y los paí- 
ses disminuirán los costos de atención de estos problemas en las comunidades, principio particularmente importante en el desarrollo del país (33).

Mejorar las habilidades de crianza: la organización e implementación de talleres y grupos de padres cuyo objetivo sea este tendrá resultados positivos para la reducción de trastornos mentales, en especial del consumo de sustancias, pues es una intervención muy efectiva (34).

Identificación de factores de riesgo en individuo, familia y comunidad: las relaciones familiares, los acontecimientos vitales estresantes, la psicopatología de los padres y estilos de crianza han sido ligados a un aumento de los comportamientos desafiantes (35).

Optimizar los factores ambientales: donde se desarrolla el niño y los estilos de crianza como factor de protección mental $(36,37)$.

Las enfermeras domiciliarias deben filtrar y derivar a las familias y pacientes con factores de riesgo para recibir una prevención o intervención selectiva e indicada (38). Entre las intervenciones más reconocidas se encuentran: The Triple P: A Multilevel Parenting Intervention, creada por Sanders y Markie-Dadds en el 2000 (39); The Stregthening Families Program (SFP) (40), o intervenciones combinadas de mejora de crianza y escuelas como The Incredible Years Program, realizado en 1990 por Webster-Stratton (41).

\section{Limitaciones}

El presente estudio posee algunas limitaciones, como el tamaño muestral reducido, que pudo haber influido en la disminución del poder estadístico para la identificación de factores de riesgo. Además, las mediciones diagnósticas del grupo clínico fueron con base en el sistema de Clasificación Internacional de Enfermedades, asignado por el clínico y no mediante un instrumento observacional o estandarizado. El estudio de los factores de riesgo biológico-individuales (pre, peri y posnatales) fue retrospectivo. Idealmente, debiera contemplarse un estudio longitudinal y prospectivo.

\section{Conclusiones}

Por todo lo descrito, se hace hincapié en la relevancia y la necesidad de orientación a una detección temprana de los factores de riesgo que varían según la dimensión: biológico individual, parental y contextual, por lo que estos factores deben abordarse en las primeras etapas de la vida, realizando programas que incluyan intervenciones en los hogares durante el embarazo y la infancia temprana, dirigidas a poblaciones vulnerables, especialmente a niños que provienen de familias de bajos ingresos y niveles educativos, al darles a los padres capacitación sobre las prácticas efectivas de crianza de los hijos en conjunto con las escuelas en preescolar y primaria.

La identificación oportuna de niños con factores de riesgo en su desarrollo permitiría orientar, gestionar e iniciar acciones específicas de ayuda; así como alcanzar mejores resultados en la evolución del desarrollo 
infantil, ganancias de calidad de vida en cuanto se refiere de forma eficaz a una intervención oportuna, mejoras en la satisfacción de los usuarios y aumento de la eficiencia del sistema de salud, por una disminución en los costos, trabajo y tiempo.

Además, ello desempeñar un buen papel de filtro, al aplicar cuestionarios de tamizaje y proporcionar la información y educación necesaria a los futuros padres y a los que ya lo son, para que sean ellos los principales detectores de estos factores que pueden causar una desviación en el desarrollo normal de sus hijos; así como ajustando las derivaciones a especialistas y pruebas complementarias de los niños que reporten factores de riesgo y que comprometan de forma directa la calidad de vida de ellos y sus familias.

\section{Referencias}

1. Acle TG, Roque HMP, Zacatelco RF, Lozada GR, Martínez BCM. Discapacidad y rezago escolar: riesgos actuales. Act Colom Psicol [internet]. 2007 [citado 18 septiembre 2013];10(2):19-30. Disponible en: http://www.scielo.org.co/scielo.php?script=sci_arttext\&pid =S0123-91552007000200003.

2. World Health Organization. Mental health policy and service guidance package: Child and adolescent mental health policies and plans. Ginebra; 2005.

3. Santangelo SL, Pauls DL, Goldstein JM, Faraone SV, Tsuang MT, Leckman JF. Tourette's syndrome: what are the influences of gender and comorbid obsessive-compulsive disorder? J Am Acad Child Adolesc Psychiatry. 1994;33(6):795-804.

4. Vasconcelos MS, Sampaio AS, Hounie AG, Akkerman F, Curi M, Lopes AC, Miguel EC. Prenatal, perinatal, and postnatal risk factors in obsessive-compulsive disorder. Biol Psychiatry. 2007;61(3):301-7.

5. Albores G, Márquez C, Estañol B. ¿Qué es el temperamento?: el retorno de un concepto ancestral. Salud Mental. 2003;26(3):16-26.

6. Valencia GMR. El control y la resiliencia del yo en los problemas de los niños y niñas. Tesis de doctorado no publicada. Facultad de Psicología, Universidad Nacional Autónoma de México; 2005.

7. Betancourt-Ocampo D, Andrade-Palos P. La influencia del temperamento en problemas internalizados y externalizados en niños. Revista Intercontinental de Psicología y Educación. 2008;10:29-48.

8. Caraveo A, Nicolini H, Villalba A, Wagner F. Psicopatología en familiares de tres generaciones:un estudio epidemiológico en la Ciudad de México. Salud Pública de México. 2005;47(1):23-29.

9. Gottlieb G. Probabilistic epigenesist. Dev Sci. 2007;10(1):1-11.

10. Cicchetti D, Garmezy N. Risk and protective factor in the development and psychopathology. Cambridge: Cambridge University Press; 1993. 
11. Zeanah $\mathrm{CHH}$, Boris $\mathrm{NW}$, Larrieu JA. Infant development and developmental risk: A review of the past 10 years. J Am Acad Child Adolesc Psychiatry. 1997;36(2):165-78.

12. Jadue JG. Transformaciones familiares en Chile: riesgo creciente para el desarrollo emocional, psicosocial y la educación de los hijos. Estud Pedagóg [internet]. 2003;29:115-26. Disponible en: http:// mingaonline.uach.cl/scielo.php?pid=s071807052003000100008\&sc ript=sci_arttext.

13. Espin JJC, Belloso RJJ. Estudio descriptivo de los niños y adolescentes nuevos vistos en un servicio de salud mental en el periodo de un año. Rev Asoc Esp Neuropsiq. 2009;29(104):329-54.

14. Medina M, Borges G. Prevalencia de trastornos mentales y uso de servicios: resultados de la Encuesta Nacional de Epidemiología Psiquiátrica en México. Salud Mental. 2003;26(4):2.

15. Márquez CME, Zanabria SM, Pérez BV, Aguirre GE, Arciniega BL, Galván GCS. Epidemiología y manejo integral de la discapacidad intelectual. Salud Mental. 2011;5(34):443-9.

16. World Health Organization (WHO). Atlas: Global resources for persons with intellectual disabilities 2007. Geneva; 2007.

17. Márquez CME, Zanabria SM, Diaz PJA, Arciniega BL et al. Guía de retraso mental. En: Ulloa R, Fernández C, Gómez H, Ramírez J, Reséndiz J, editores. Guías clínicas: Hospital Psiquiátrico Infantil "Dr. Juan N. Navarro" [internet]. México D. F.; 2010 [citado 28 ago 2012];239-65. Disponible en: http://www.sap.salud.gob.mx/downloads/pdf/nav_guias9.pdf\&embedded=true\&chrome=true.

18. Caraveo AJ, Colmenares BE, Martínez VN. Síntomas, percepción y demanda de atención en salud mental en niños y adolescentes de la Ciudad de México. Salud Pública México. 2002;44:492-8.

19. Valencia GMR. El control y la resiliencia del yo en los problemas de los niños y niñas. Tesis de doctorado no publicada. Facultad de Psicología, Universidad Nacional Autónoma de México; 2005.

20. Nogales IIA, Ulloa FRE, Rodríguez JH, Palacios CL, Vázquez MJ, Munguía MA et al. Depresión en niños y adolescentes. En: Guías clinicas: Hospital Psiquiátrico Infantil Juan N. Navarro. México, D. F.: Secretaría de Salud; 2010. p. 65-85.

21. Esparza N, Rodríguez MC. Factores contextuales del desarrollo infantil y su relación con los estados de ansiedad y depresión. Revista Diversitas-Perspectivas en Psicología. 2009;5(1):47-65.

22. Caraveo A. Cuestionario breve de tamizaje y diagnóstico de problemas de salud mental en niños y adolescentes: algoritmos para síndromes y su prevalencia en la Ciudad de México. Segunda parte. Salud Mental. 2007;30(1):48-55.

23. Fernández BFJ. Estrés, riesgo familiar e inadaptación socioemocional y escolar en la infancia. Tesis doctoral, Facultad de Psicología, Málaga, España; 2007. 
24. Antolín L, Oliva A, Arranz FE. Contexto familiar y conducta antisocial infantil. Anuario de Psicología. 2009;40(3):313-27.

25. Leckman JF, Hardin MT, Dolansky ES, etal. Perinatal factors in the expressions of Tourette's Syndrome. J Am Acad Child Adolesc Psychiatry. 1990;29: 220-26.

26. World Health Organization. Mental health atlas. Geneva; 2005.

27. Márquez CME, Hernández GL, Aguilar VJ, Pérez BV, Reyes SM. Datos psicométricos del EMBU-I "Mis memorias de crianza" como indicador de la percepción de crianza en una muestra de adolescentes de la Ciudad de México. Salud Mental. 2007;30(2):58-66.

28. Walker SP, Wachs TD, Grantham-McGregor S, Black MM, Nelson CA, Huffman SL, Baker-Henningham H, Chang SM, Hamadani JD, Lozoff B, Gardner JL, Powll CA, Atif R, Richter L. Inequality in early childhood: risk and protective factors for early child development. Lancet. 2011 DOI: 10.1016/S0140-6736(11)60555-2.

29. Repetti LR, Taylor ES, Seeman TE. Risky families: Family social environments and the mental and physical health of offspring. Psychol Bull. 2002;128:330-66.

30. Salazar TI, Varela AMT, Tovar CJR, Cáceres RE. Construcción y validación de un cuestionario de factores de riesgo y de protección para el consumo de drogas en jóvenes universitarios. Acta Colombiana de Psicología. 2006;9(2):19-30.

31. Organización Panamericana de la Salud, Organización Mundial de la Salud, Secretaría de Salud. Informe sobre sistema de salud mental en México. México; 2011.

32. O'Connell ME. Preventing mental, emotional, and behavioral disorders among young people: Progress and possibilities. Washington DC: National Academies Press; 2009.

33. Avan B, Richter L, Ramchandani P, Norris S, Stein A. Maternal postnatal depression and children's growth and behaviour during the early years of life: exploring the interaction between physical and mental health. Arch Dis Child. 2010 Sep;95(9):690-5.

34. Barlow J, Smailagic N, Ferriter M, Bennett C, Jones H. Group-based parent-training programmes for improving emotional and behavioural adjustment in children form birth to three years old. Cochrane Database Syst Rev. 2010;(3):CD003680.

35. Einfeld S, Tonge B, Clarke K. Prevention and early intervention for behaviour problems in children with developmental disabilities. Curr Opin Psychiatry. 2013;26(3):263-9.

36. Bayer J, Hiscock H, Scalzo K, Mathers M, McDonald M, Morris A, Birdseye J, Wake M. Systematic review of preventive interventions for children's mental health: what would work in Australian contexts? Aust N Z J Psychiatry. 2009;43(8):695-710.

37. Sieh D, Meijer A, Oort F, Visser-Meily J, Van der Leij D. Problem behavior in children of chronically ill parents: a meta-analysis. Clin Child Fam Psychol Rev. 2010;13(4):384-97. 
38. Li L, Liu J. The effect of pediatric traumatic brain injury on behavioral outcomes: a systematic review. Dev Med Child Neurol. 2013 Jan;55(1):37-45.

39. Sanders M, Markie DC, Tully L, Bor W. The Triple P-Positive Parenting Program: A comparison of enhanced, standard, and selfdirected behavioral family intervention for parents of children with early onset conduct problems. J Consult Clin Psychol [internet]. 2000;68(4):624-40. Disponible en: de:http://www.prevention.psu. edu/news/documents/Sanders_Markie-Dadds_Tully_Bor_2000.pdf.

40. Kumpfer KL, Alvarado R. Family-strengthening approaches for the prevention of youth problem behaviors. Am Psychol. 2003;58(67):457-65.

41. Webster SC, Reid M, Stoolmiller M. Preventing conduct problems and improving school readiness: Evaluation of the incredible year's teacher and child training programs in high-risk schools. J Child Psychol Psychiatry. 2000;288(15):471-88. 
